\title{
Mental Files, Blown up by Indexed Files*
}

\author{
Isidora Stojanovic \& Neftalí Villanueva Fernández
}

\begin{abstract}
Our paper discusses Recanati's application of the mental files apparatus to reports of beliefs and other attitudes. While mental files appear in Recanati's work on belief-reports early on (e.g. Recanati 1993), Recanati's latest book introduces the concept of indexed files (a.k.a. vicarious files) and puts it to work to explain how we can report other people's attitudes and to account for opacity phenomena. Our goal is twofold: we shall show that the approach in Recanati 2012 departs significantly from his earlier proposals (1993, 2000) as well as from the very spirit of truth-conditional pragmatics (2010), and we shall argue that the indexed files approach, qua an attempt to provide a semantics for beliefreports, is untenable.
\end{abstract}

\section{Sect. 1: Introduction: from Modes of Presentation to Mental Files}

Recanati has dealt with belief-reports and, more generally, attitude ascriptions in a number of places over the past 20 years. Some of this work shows important similarities with the framework presented in 2012, but there are significant divergences as well. We shall review the ideas already present in Recanati's earlier work $(1993,2000)$, introduce his new approach, and then point out certain features of the latter that make it difficult to draw a homogeneous picture of Recanati's views about metarepresentation; that is, about the capacity of thinking about attitudes - be they other people's or our own - and of reporting them.

In Direct Reference (Recanati 1993), one of Recanati's main achievements was to provide convincing arguments against the so-called 'implicature theory' of belief-reports (cf. Salmon 1986) and offer an alternative on which pragmatic processes affect the truth value of the belief-report, without giving up the Direct Reference view of singular terms. In a nutshell, the proposal was that singular terms in embedded that-clauses contribute their reference, and nothing but their reference, to the proposition expressed. However, opaque attributions, characterized by the fact that co-referential terms cannot be intersubstituted salva veritate, give rise to an optional pragmatic process by means of which some particular mode of presentation is contributed to the proposition expressed, and this contextually contributed mode of presentation is responsible for the lack of substitutivity. The pragmatic process at stake, quasi-singularization (cf. 1993: 354) affects the proposition expressed by the entire belief attribution, but is truth-conditionally irrelevant with respect to the truth-conditions of the embedded that-clause. On the other hand, the proposition expressed in the case of transparent attributions makes no appeal to any specific mode of presentation. To illustrate, consider the sentence:

\section{(1) Zvetlana believes that Norma Jean Mortenson was a happy person.}

What is at issue is how the context in which the sentence is uttered may affect the truth conditions of the report. Let us first consider a context in which both the speaker and the hearer know that Norma Jean Mortenson is Marylin Monroe, and it is common knowledge amongst them that Zvetlana knows it, too. Then, typically, the report should be read as an example of a transparent report, since there is no reason to suppose that the particular name that the speaker is using to refer to the individual Norma Jean Mortenson / Marylin

\footnotetext{
${ }^{*}$ Published in Inquiry 58 (2014): 1-15; for citations, please refer to the published version
} 
Monroe is of any relevance to what the speaker is saying or even communicating by her utterance. In such an example, 'Norma Jean Mortenson' and 'Marylin Monroe' may be intersubstituted salva veritate.

Now, compare this with a context in which Zvetlana, a school friend of Norma Jean Mortenson, does not know realize that her school friend is the actress Marylin Monroe. Zvetlana believes of her school friend that she was a happy person, while at the same time believing that the famous actress, about whom she heard from newspapers, was not a happy person. If this is known to the speaker and the hearer, then the sentence in (1), uttered in such a context, should be given an opaque reading. For, substituting the name 'Marylin Monroe' to 'Norma Jean Mortenson' would take us from a true report to a false report, hence subsitutivity fails. On (Recanati 1993)'s analysis, what accounts for the failure of subsitutivity is that a particular mode of presentation is pragmatically contributed to the proposition expressed. Whether the speaker uses the one name or the other would make no difference to the semantic contribution of the name (in either case, the name contributes its reference, i.e. the individual Norma Jean Mortenson / Marylin Monroe), but it would make a difference to the pragmatic processes that, in turn, affect the truth value of the overall report.

Recanati's indexed files approach (which we shall explain shortly) follows the broad lines of his 1993 approach, which itself broadly follows the proposal set out in (Crimmins and Perry 1989). As before, the singular term contributes its reference, and nothing but its reference, to the semantic content, whereas what accounts for opacity phenomena is the way in which the ascribee thinks of the reference. This "mode of presentation" is, in Recanati's current proposal, cashed out in terms of a mental file. It is a special kind of file, namely, a "vicarious" file: a file that belongs among the ascriber's mental files, but stands for how the ascribee thinks of the referent of the file (assuming there is one); it is thus a mental file "indexed" to someone other than the owner of the file. As he puts it:

To account for the vicarious use of files, we need the notion of an indexed file. An indexed file is a file that stands, in a subject's mind, for another subject's file about an object. An indexed file consists of a file and an index, where the index refers to the other subject whose own file the indexed file stands for or simulates. (2012: 183)

The idea of vicarious files is fairly intuitive. Consider Zvetlana, from our example, who thinks of Norma Jean Mortenson (that is, of Marylin Monroe) in terms of her childhood acquaintance and without realizing that she became a famous actress and ended her life tragically. We, who are aware of this, can think of Marylin Monroe by means of our own respective files (e.g. picturing her as an attractive blond woman in a red dress, or whatever), but we can also think of her vicariously using Zvetlana's mental file, e.g. imagining her as a shy school girl who would become an ordinary person and lead a happy and peaceful life.

Now, how do vicarious files make their way into an account of metarepresentation, of attitude ascriptions, and of opacity phenomena? Although (Recanati 2012) does not go all that far in spelling out the details of the proposal, we can reconstruct the general idea in the following way. Consider an opaque reading of the belief-report in (1); that is, a reading in which substituting 'Marylin Monroe' to 'Norma Jean Mortenson' would turn the report from a true one to a false one. The name 'Norma Jean Mortenson' contributes its referent, viz. Marylin Monroe, to the proposition expressed, and the way this referent is determined is by its being the referent of the reporter's mental file associated with the name. But at the same time, the context somehow contributes an indexed file to the proposition expressed 
by (1), namely, a file about Norma Jean Mortenson / Marylin Monroe that is indexed to the reportee, i.e. to Zvetlana. And given that Zvetlana has two files for Norma / Marylin, a "Norma Jean Mortenson" file as well as a "Marylin Monroe" file, and that the two files are not only distinct but not even "linked" ${ }^{1}$, substituting one name with another name, albeit coreferential, will make a different file contextually salient, resulting in the contribution of a different constituent - viz. a different indexed file - to the truth-conditional content of the belief-report.

Although the above outlined account of opacity and of the failures of subsitutivity of coreferential names in belief-reports may strike one as somewhat sketchy, this is pretty much all that one gets from the text. We prefer to leave it sketchy and abstrain from attempting to fill in any formal details that one would ideally ask for in order to understand how a given sentence, uttered in a given context, gets to be assigned one truth-conditional content rather than some other. Now that we have outlined an application of the indexed files proposal to opaque belief-reports, let us turn to its account of transparent reports in the next two sections.

\section{Sect. 2: A Hidden Variable for the Indexed File?}

Our goal in this section is to show how Recanati's 2012 clashes with certain ideas that he has fiercely defended elsewhere $(2000,2010)$ regarding the nature of the processes that contribute a mode of presentation (or, as the case may be, an indexed file) to the proposition expressed by a given belief attribution.

In Oratio Obliqua, Oratio Recta (2000), Recanati uses belief attributions as a case-study to support a more general view regarding our language architecture, a view known as TruthConditional Pragmatics. In a nutshell, what characterizes such "radically contextualist" views is the idea that genuinely pragmatic processes that are not triggered by any syntactic or semantic element may contribute truth-conditionally relevant ingredients to the proposition expressed and thus affect the truth value of an utterance (see Recanati 2004). The view, or the family of views, that Recanati's contextualism was confronting, are characterized by the assumption that any component of the proposition can be traced back to the logical form of the sentences, and that it is either directly provided by the linguistic meaning of an expression or else "recruited" from the context as a result of interpreting some context-dependent expression present in the logical form (hence as a result of what Recanati calls a bottom-up primary pragmatic process). Recanati's TruthConditional Pragmatics, on the contrary, hinges on the existence of top-down primary pragmatic processes, which result in including into the proposition expressed even context-dependent information for which there is no linguistic trace at the level of the logical form of the sentence uttered, whether superficial or deep. One of the key achievements in (Recanati 2000) was to offer an account of metarepresentations as a case-study that supports Truth-Conditional Pragmatics. Opacity was seen as a genuinely pragmatic phenomenon that can affect the truth-conditions of belief-reports, blocking substutivity of co-referential singular terms in embedded 'that'-clauses.

The two main rival theories of opacity confronted in (Recanati 2000) were, on the one hand, the implicature theory (Salmon 1986, but also Saul 1998) and, on the other, the hidden-indexical theory (Crimmins and Perry 1989, Schiffer 1992, Crimmins 1995). According to the former, modes of presentation do not impact the semantics of belief attributions; however, they systematically generate conversational implicatures. Thus, to return once more to the example in (1), the speaker says that Zvetlana believed that the

1 Throughout Recanati 2012, several concepts of "linking" relations between files are developed; see pp. 42-53, 82-88, 94-99, 183-6, 191-6. 
individual Norma Jean Mortenson / Marylin Monroe was a happy person, and implicates that Zvetlana was thinking about this individual by means of a certain mode of presentation associated with the name 'Norma Jean Mortenson'. According to the latter, modes of presentation are full-fledged constituents of the proposition that a given beliefreport expresses, and are contributed to this proposition via a process of saturation, i.e. an assigment of contextually specified values to a hidden indexical variable. Both for the implicature theorists and the hidden-indexical theorists, belief-reports systematically involve modes of presentation; the difference between the two views lies in the process via which the specific mode of presentation required in a particular situation is reached, namely conversational implicatures vs. saturation.

Against the implicature theory, Recanati held that modes of presentation were needed to account for the truth-conditions of opaque belief-reports, rather than showing up merely at the truth-conditionally inert level of conversational implicatures. Against the hiddenindexical view, he held that belief-reports do not systematically recruit a specific mode of presentation to be contributed to the proposition expressed. Rather, 'believe' and other attitude verbs were seen as, in a way, ambiguous expressions that could be used to express a dyadic operator, specifying two syntactic arguments - one for the attitude-holder and the other, for the propositional content of the attitude -, or a triadic operator that in addition specifies an argument-slot for the mode(s) of presentation. ${ }^{2}$ Crucially, transparent belief-reports were seen as not involving any mode of presentation - neither some kind of neutral, generic or arbitrary mode of presentation, nor an existential quantification over modes of presentations. It is precisely the rejection of the idea that transparent reports involve an implicit existential quantification over modes of presentation that allowed Recanati's 2000 framework to be clearly distinguished from the hidden-indexical view and to foster his defense of (radical) contextualism more generally. Whereas for the hidden indexical theory, the transparent vs. opaque distinction boils down to whether the implicit argument for a mode of presentation is existentially quantified over or has been assigned some specific mode of presentation as its value, for (Recanati 2000), it correlates with the issue of whether the meaning of the sentence involved in a belief-report undergoes a genuinely pragmatic, or "free", enrichment. As he writes:

The transparent/opaque ambiguity for belief-reports is therefore an ambiguity between the minimal reading and a contextually enriched reading of the sentence (2000: 159).

Now, the reader of (Recanati 2012) familiar with his earlier work on opacity may be somewhat surprised (to put it mildly) to discover that in his current view, not only do mental files play the role of modes of presentation, but also that some key aspects of his earlier proposal are no longer to be found. In particular, transparent reports are now also seen as involving modes of presentations, albeit existentially quantified over:

In transparent attitude ascriptions, [...] there is implicit existential quantification over the modes of presentation (mental files) in the ascribee's mind. (2012: 185).

Some might think that whether transparent ascriptions do not involve any mode of presentation or instead have the argument for the mode of presentation existentially quantified over amounts to a distinction without a difference. Indeed, on the assumption that a subject's thought is necessarily mediated by some mode of presentation or another, one might expect to find some form of equivalence between an account of tranparent

2 It should be emphasized that this idea was already outlined in Barwise (1989: 241). See also Villanueva $(2005,2006)$ and Jaszczolt $(2007)$ for elaborations on this sort of view. 
belief-reports that simply construes the belief relation as a dyadic one (between the subject and the content of his or her belief) and an account that construes the belief relation as a three-place one (including, in addition, a slot for a mode of presentation) but then existentially quantifies over this third argument when it comes to transparent reports.

However, it should be stressed that the two accounts are not mere notational variants. There are important differences between the two types of account that raise issues regarding the syntax-semantics interface as well as the semantics-pragmatics interface. What is more, they raise precisely the sort of issues that have been lying at the heart of Recanati's previous work $(2004,2010)$ and have generated some fierce debates between "contextualists" and "minimalists". ${ }^{3}$ In particular, to defend radical contextualism, it was crucial to establish that the contribution of a mode of presentation to the truth-conditional content of an opaque belief-report was the result of a pragmatic process such as freeenrichment that is not to be traced to the presence of some implicit argument, some hidden variable, that was already there in the logical form of the report. Recanati's latest proposal on which in transparent reports, "there is an implicit existential quantification over the modes of presentation (mental files)" (ibid.) is thus truly surprising and may be seen as a concession to his opponents, who hold that "all effects of extra-linguistic context are traceable to elements in the actual syntactic structure of the sentence uttered" (Stanley 2000: 391).

\section{Sect. 3: Opacity everywhere}

As we have seen in the previous section, Recanati's most recent take on the nature of belief-reports does not seem to suit his overall theoretical enterprise. The presence of implicit existential quantification over modes of presentation even in transparent reports makes it impossible to treat belief-reports as a case-study for Truth-Conditional Pragmatics. We turn now to another point where Recanati seems to have drastically departed from his old views on the subject. Within the framework of Truth-Conditional Pragmatics, opaque belief-reports containing singular terms express 'enriched' propositions, singular propositions plus a mode of presentation. Transparent reports, on the other hand, embed just singular propositions. Opaque reports thus require the use of further cognitive resources on the part of the speaker and the audience in order to convey and understand a larger amount of information. This cognitive requirement thus makes transparent reports the most suitable candidate to serve as a default reading for belief ascriptions. Now, Recanati appears to approach the matter completely differently now. The purpose of this section is to explore both the reasons that have lead Recanati to change his theoretical stance and the problems that his new stance needs to face.

In (Recanati 2000a) he explicitly takes sides with those who defend the default, or more fundamental, nature of transparent belief-reports:

The view I have just expounded and ascribed to Frege, Russell, and Quine deserves to be called the Classical View. It has been enormously influential. As a result it is now commonly accepted that metarepresentations are opaque, and that that is so because the object-representation is mentioned rather than used. In the first two parts of this book I have tried to go as far as possible in the opposite direction. Metarepresentations, I claimed, are fundamentally transparent. (2000a: 114).

Recanati seemed to be close to the views of Jaszczolt, who defended the theoretical pertinence of a default de re principle (cf. Jaszczolt 1999: 121 and ff.). As she writes:

3 For a survey of the issues at the heart of those debates and for discussion, see Stojanovic (2008). 
The de re reading of sentences ascribing beliefs is the default reading. Other readings constitute degrees of departure from the default, arranged on the scale of the strength of intentionality of the corresponding mental state. (1999: 190).

On the other side of the spectrum, certain analyses were committed to the dominant status of the opaque reading for belief-reports. Recanati summarizes these attempts -and rejects them- while discussing the implicature theory (Recanati 1993: 325 and ff.). The implicature theorist, as Recanati reconstructs this position, takes advantage of a new Gricean maxim, the maxim of faithfulness, which urges the speaker to try to be 'faithful to the believer's own point of view, unless there are reasons not to do so' (Recanati 1993: 333). Recanati provided several arguments against this view, as early as in 1993, and dismissed it, together with the default nature of the opaque reading.

The mental-files approach to belief-reports has taken Recanati in a different direction. "It is a characteristic of attitude reports" he says, "that often, the words in the embedded clause are associated with the ascribee's mental files rather than, or in addition to, the speaker's own files. This corresponds to the opaque reading of such reports." (Recanati 2012: 182). belief-reports "typically involve two modes of presentation: the speaker's (a regular file) and the ascribee's (an indexed file)" (191), and transparent cases "if they exist, are marginal" (loc. cit.).

Before we address the question of why Recanati thinks that transparent belief-reports are marginal (if they exist at all!), we must clarify a terminological issue. Recanati was one of the first to point out that Quine's 1956 distinction between two senses of believing, usually called the de re vs. de dicto distinction, needed to be unpacked into two orthogonal distinctions: relational vs. notional, and transparent vs. opaque (cf. Recanati 2000b). These two distinctions respond to different criteria, exportation and substitutivity. Singular terms contained in relational belief-reports can be "exported"; that is to say, an existential statement can be deduced from such reports. If "There is someone whom Zvetlana believes to be a happy person" can be inferred from (1) -Zvetlana believes that Norma Jean Mortenson was a happy person-, then (1) is a relational belief ascription. If the singular term 'Norma Jean Mortenson' cannot warrant the inference to the corresponding existential statement, then the belief-report would be notional - it would be a "freewheeling case", in Recanati's current terminology.

Transparency and opacity, on the other hand, depend on substitutivity. belief-reports seem to be a particularly hospitable context for substitutivity problems, cases where perfectly natural substitutions of one singular term for a different one with the same meaning appear to be blocked. Substitutivity can be blocked for co-extensional expressions in many different kinds of contexts, such as those that involve temporal operators. Thus 'Obama' and 'the President of the US' cannot be intersubstituted salva veritate in "Twelve years ago, the President of the US decided to attack Afghanistan". Even though these substitutions are often blocked within belief-reports, only co-intensional substitutivity problems are seen as genuinely characteristic of opaque belief-reports. A belief-report will be opaque if the singular terms contained in it cannot be substituted by other cointensional singular terms salva veritate. In transparent belief-reports, co-intensional expressions can be de iure intersubstituted salva veritate. Recanati has been advocating these distinctions for years, and we have no reason to suppose that they should be used differently in his most recent writings.

(Recanati 2012)'s argument for the dominance of opaque readings is based on the systematic presence of two modes of presentation in belief-reports. Any belief-report, Recanati claims, involves the speaker's regular file, and the ascribee's indexed file. Even 
in cases where this does not appear to be so, Recanati says, "this does not prevent the ascribee's file from being contextually recoverable to some extent, and to affect the truthconditions of the report" (2012: 192). So, even in a context in which the speaker was totally unaware that a single person could mistake the identity of 'Norma Jean Mortenson' and 'Marylin Monroe' -a classical transparent context- and (1) was uttered, the substitution of one singular term for the other could create a new context where the ascribee's mode of presentation could be recoverable, and affect the truth conditions. Cointensional substitutivity will be blocked, and thus we would have no reason to consider the ascription as transparent. Since Recanati takes this to be a pervasive phenomenon, he concludes that transparent reports are marginal.

In (Recanati 2000a), a different strategy is adopted to deal with this very same phenomenon. Recanati recognizes that substitutivity of co-intensional terms salva veritate is generally blocked in belief-reports. Nevertheless, only opaque reports are taken to involve the ascribee's mode of presentation. Transparent reports are not "enriched" with the ascribee's mode of presentation, they are purely referential. In opaque reports, the context is "reflecting"; in transparent reports, a potential substitution can make it "reflecting", and so substitutivity is blocked for both opaque and transparent attributions (2000a: 161). Crucially, this does not prevent Recanati from recognizing the existence, and dominance, of transparent belief-reports: "oratio obliqua per se is not quotational. In its pure form, oratio obliqua is transparent. Opaque oratio obliqua is a more complex phenomenon: it involves some measure of quotation in addition to the basic pattern" (2000a: 207). So, even though singular terms cannot be de facto intersubstituted in beliefreports, these reports will be transparent because, in the particular context in which they are uttered, their truth conditions may be specified without taking into consideration the ascribee's mode of presentation. Whatever happens to these transparent ascriptions when the substitution is performed, and when they are subsequently transposed into a different context, is irrelevant to assessing their original transparency. ${ }^{4}$ In (Recanati 2012), on the other hand, further substitutivity problems are used to determine that the ascribee's mode of presentation was present in the original belief-report, and so we have no reason to consider it transparent.

As we saw in the previous section, the dominance of the transparent reading was an asset to Recanati's Truth-Conditional Pragmatics. We think, though, that it is an idea worth pursuing on its own. Opaque belief-reports, as Recanati himself pointed out (cf. 2000a: 207), are a complex phenomenon, and this complexity may render the analysis of beliefreports unmanageable if we take the opaque reading to be the default reading for belief ascriptions. Our line of reasoning here requires two steps: firstly, iterated belief-reports could not be understood on a regular basis unless there was a default preference for one of the options, transparent or opaque; secondly, it is reasonable to suppose that as we iterate belief operators, this increases the likelihood of a transparent reading. Let us illustrate the point with some examples (which, admittedly, may require some effort to process):

(2) Lana Lang believes that Batman believes that Superman can fly.

(3) Lana Lang believes that Spiderman believes that Batman believes that Superman is an oculist.

Let's take a quick look at the epistemic possibilities that might determine the modes of presentation involved in these ascriptions. Lana Lang may be aware/unaware that Batman

\footnotetext{
4 Incidentally, this argument, apparently rejected in (Recanati 2012), is formally identical to the one used in (Recanati 2002) to defend genuine unarticulated constituents from Stanley's binding argument. The fact that a change of context can make a genuine unarticulated constituent appear in a given proposition does not prove, Recanati argued then, that the unarticulated constituent was always there.
} 
is Bruce Wayne, she may be aware/unaware that Superman is Clark Kent, and she may be aware/unaware that Batman/Bruce Wayne is aware/unaware that Superman is Clark Kent. With a couple more of expressions susceptible to be interpreted as opaque, 'Spiderman' and 'being an oculist', the number of possibilities increases spectacularly. The amount of information needed to favor one of these interpretations and exclude the others is huge. It would be absurd to suppose that every speaker willing to say (2) or (3) is able to manipulate enough contextual features so as to be confident that the audience can appropriately grasp the correct option. We think that the most reasonable way to accommodate nested belief reports is to refuse to go along with (Recanati 2012)'s contention that the opaque interpretation is the default one for belief-reports, and to hold onto the mainstream, more conservative view, according to which in the interpretation of attitude ascriptions, the transparent reading is the norm, while the opaque readings are the exception. $^{5}$

\section{Sect. 4: Indexed Files ex Machina}

Ascribing mental attitudes to other people, or to oneself, and reporting them, have been areas in philosophy of language that hosted a series of puzzles and problems to which, up to our days, no adequate solution has arguably been proposed and accepted widely. They are difficult areas because they involve an array of problems, having to do with the nature of beliefs (and other attitudes) and requiring an understanding of metarepresentational capacities in humans, over which theories in psychology, philosophy of mind and cognitive science have been stumbling for decades. Recanati's frameworks of mental files certainly offers interesting and novel perspectives over these issues, and we leave it to theorists concerned with the nature of metarepresentation and to philosophers of mind to evaluate its merits and its pitfalls. Our own focus has been on a language-related topic, namely, on the application of the mental files framework to the semantics of attitude ascriptions. We have been guided by two goals: first, discuss the similarities and the differences between Recanati's earlier proposals and his current approach and, second, point out two features of his current approach that we see as a regress, rather than progress, with respect to his earlier views. The two features at stake both have to do with transparent reports. The first lies in the idea that transparent reports involve "an implicit existential quantification over the modes of presentation (mental files)" (185) and the second, in the idea that such reports "if they exist, are marginal" (191). In sections 2 and 3 we discussed the two ideas respectively. We pointed out that the first one generates a major clash with Recanati's enterprise of Truth-Conditional Pragmatics, and we argued that the second not only clashes with Recanati's earlier view on the matter, but is empirically implausible and squares badly with the possibility of iterated belief-reports. Our goal in the last section is to shed some further doubts on Recanati's proposal, qua an attempt to understanding the semantics and the pragmatics of attitude ascriptions.

Philosophers of language and semanticists have been attempting over years to put forward formal (or at least, reasonably formal) proposals regarding the interpretation of belief-reports. The question, to put it as simply as possible, is to explain how a sentence of the form 'NP believes that S', as used in such-and-such context, may, or should, be assigned such-and-such truth value; and similarly for other attitude verbs ('desires', 'regrets', etc.). Now, the proposals sought should be general enough so that NP can be occupied by any noun phrase (hence quantified phrases such as 'everyone' or 'most druck-drivers', and not only proper names such as 'Zvetlana' or definite descriptions), and so that the analysis may extend compositionally to belief-reports occurring within more complex sentences (such as "If Ahmed believes that his mother doesn't love him, then he is unhappy", or "Nobody thinks that it is possible that Ahmed believes that his mother

5 Our view largely thus agrees with the one defended in Taylor (2007). 
doesn't love him"). This, in a nutshell, is the sort of questions that accounts of attitude ascriptions, whether semantic or philosophical, have been aiming to answer ever since the pioneering work of Frege's Sinn und Bedeutung.

In light of this clarification, it becomes particularly difficult to be critical of (Recanati 2012)'s proposal, because the basic answers that the above questions call for are simply missing. Whether indexed files may or may not turn out to be useful tools in providing an account of attitude reports is impossible to evaluate in the absence of such basic elements of the proposal. (Recanati 2012) merely offers a discussion of a couple of specific cases. What is more, the discussion, for one thing, omits all crucial detail and, for another, fails to generalize. Thus Recanati invites us to "consider the possible interpretations of an attitude report of the form ' $x$ believes that $a$ is $F^{\prime}(2012$ : 185) but explicitly restricts the discussion to the cases "where ' $a$ ' is a genuine singular term" and implicitly restricts it to the cases where ' $x$ ', too, is a singular term. He suggests (186) that in "standard opaque attributions" (...) "two files are potentially relevant to the interpretation of the utterance, one provides the speaker's own way of thinking of the referent, and the other the ascribee's way of thinking". How do these files find their way into the interpretation of the utterance? Once more, we are not given a clear and straight answer. Rather, "to see how the two modes of presentation come into play", Recanati invites us to consider a very elaborate example of an opaque attribution, whose ultimate aim is to conclude that both files are "relevant to the semantic content of the utterance" (188, our italics). His reasons for concluding this is, on the one hand, that "the ascribed [ascribee's] mode of presentation pertains to the semantic content because it is truth-conditionally relevant" (188), while "the fact that the conventional meaning of the expression constrains the file is enough (...) to make the latter relevant to the semantic interpretation of the utterance" (189).

To forestall misunderstanding, we are not disputing the claim that both files, the speaker's and the ascribee's, may be "relevant to the semantic content". In order to dispute that claim, we would need to have a clear grasp of the relevant notion of relevance. It would help to see what sort of semantic content is assigned to a belief report in a simple, toy example, and to see which kind of semantic and pragmatic mechanisms are at play in assigning the semantic content to a given sentence in a given context. Unfortunately, (Recanati 2012) leaves its readers figure out by themselves how the pieces of the proposal fit together. But that is a difficult task. Is there an argument-slot for the indexed file in the logical form of the attitude-reporting sentence, as Recanati's take on transparent readings appears to suggests, that "invites" a specific indexed file into the semantic content? Or do indexed files break into the semantic content out of their own will, like deiex-machina, whenever they are needed to deal with opacity? Is there a separate indexed file for every expression that occurs in the 'that'-clause of the attitude ascription? Or do indexed files only kick in for singular terms? And how many indexed files do we get when we look at cases in which an attitude is ascribed not only to a single, specific subject, but e.g. to a group of subjects, as in "Many philosophers believe that Montague was French"? Do we have a separate indexed file for each and every of those many philosophers? Or a single, shared file that is somehow indexed to the noun-phrase 'many philosophers'? Last but not least, when it comes to nested reports (briefly touched upon at the end of sect. 3), how is the proposal supposed to work? ${ }^{6}$ Without providing, at the very least, a clear hint at answering these questions, Recanati's new proposal in terms of indexed files can hardly

\footnotetext{
6 This may be the right place to briefly comment this intriguing "remark" from Recanati: "Indexed files are recursive: the file component of an indexed file may itself be an indexed file. Thus $S_{1}$ may think about $S_{2}$ 's way of thinking of $S_{3}$ 's way of thinking of some entity, and to that effect may entertain the indexed file $<\mathrm{f}, \mathrm{S}_{3}>$, $S_{2}$." (2012: 183) It may be speculated that his reasons for making this remark were to anticipate iterated belief-reports. However, its potential applicability to nested reports is far from obvious. Not to mention that the concept of a "recursive" file that Recanati seems to have in mind can hardly be related to what either logicians or formal semanticists mean by the property of being recursive.
} 
be seen as a serious competitor against the existing semantic and pragmatic accounts of the opacity phenomena that reign over attitude ascriptions.

\section{Acknowledgments}

We would like to thank Herman Cappelen for his invitation to contribute to this symposium, as well as his patience, and François Recanati for his stimulating work and for discussion on various occasions over the past decade. Isidora Stojanovic would like to acknowledge generous support from Marie Curie Actions, the grant agreement PIEF-GA-2011-302596, project "Lexical Meaning and Logical Inference". Neftalí Villanueva acknowledges support from the projects "Naturalismo y pragmatismo contemporáneos", HUM4099 and "Conceptos de orden superior. Una aproximación expresivista", FFI2010-15704.

\section{References}

Barwise, J. (1989). The Situation in Logic. CSLI, Stanford.

Crimmins, M. (1995). "Quasi-singular Propositions: The Semantics of belief-reports." Aristotelian Society Supplementary Volume 69: 175-209.

Crimmins, M. and Perry, J. (1989). "The Prince and the Phone Booth: Reporting Puzzling Beliefs." The Journal of Philosophy 86(12): 685-711.

Jaszczolt, K. (1999). Discourse, beliefs and intentions: semantic defaults and propositional attitude ascription. Amsterdam; New York, Elsevier.

- (2007). "Variadic function and pragmatics-rich representations of belief-reports". Journal of Pragmatics 39: 934-959.

Recanati, F. (1993) Direct Reference: From Language to Thought. Blackwell.

- (2000a). Oratio Obliqua, Oratio Recta An Essay on Metarepresentation. Cambridge, Mass; London, MIT Press.

- (2000b). "Relational belief-reports." Philosophical Studies 100 (3): 255-272.

- (2002). "Unarticulated Constituents." Linguistics and Philosophy 25: 299-345.

- (2004). Literal meaning. Cambridge; New York, Cambridge University Press.

- (2010). Truth-Conditional Pragmatics. Oxford, Oxford University Press

- (2012). Mental Files. Oxford, Oxford University Press.

Salmon, N. U. (1986). Frege's puzzle. Cambridge, Mass., MIT Press.

Saul, J. (1998). "The Pragmatics of Attitude Ascription." Philosophical Studies 92: 363-389.

Schiffer, S. (1992). "Belief Ascription." The Journal of Philosophy 89(19): 499-521.

Stanley, J. (2000). "Context and Logical Form". Linguistics and Philosophy 23: 391-434.

Stojanovic, I. (2008). "The Scope and the Subtleties of the Contextualism-Literalism-Relativism Debate". Language and Linguistics Compass 2 (2008): 1171-1188.

Taylor, K. (2007). "Misplaced modification and the illusion of opacity." in O'Rourke, M. and Washington, C. (Eds.) Situating semantics: essays on the philosophy of John Perry, Cambridge, MA: MIT Press: 215-50.

Villanueva, N. (2005). "Deferential Concepts and Opacity". In Acero and Leonardi (eds.) Facets of Concepts. Padova, II Poligrafo, 131-155.

- (2006) Ascriptions with an attitude. A study on belief-reports. Granada, Editorial de la Universidad de Granada. 